Published in final edited form as:

Epilepsia. 2013 September ; 54(0 6): 17-19. doi:10.1111/epi.12267.

\title{
Antagomirs and microRNA in status epilepticus
}

\author{
David C. Henshall \\ Department of Physiology and Medical Physics, Royal College of Surgeons in Ireland, Dublin
}

\section{Summary}

MicroRNAs are an important class of non-coding RNA which function as post-transcriptional regulators of protein levels within cells. Emerging work has revealed that status epilepticus produces select changes to microRNA levels within the brain which may impact levels of proteins involved in neuronal structure and excitability, gliosis, inflammation and apoptosis. Animal studies show that targeting microRNAs using locked nucleic acid-modified oligonucleotides ("antagomirs") can have potent effects on status epilepticus, seizure-induced neuronal death and the later emergence of recurrent spontaneous seizures. Accordingly, microRNA-based therapeutics may have potential as a future treatment of status epilepticus.

\section{Keywords \\ Epilepsy; Epileptogenesis, Hippocampal sclerosis; non-coding RNA}

\begin{abstract}
MicroRNA (miRNA) is an important class of small non-coding RNA, critical for networklevel regulation of gene expression. Biogenesis of miRNAs begins with transcription of a primary transcript (pri-miRNA), mainly from intergenic or intragenic regions of the genome, by the action of DNA polymerases (e.g. pol II). Production of the mature miRNA results from sequential actions of the RNAse III enzymes Drosha and Dicer. One strand of the mature miRNA ( 22 nucleotides) is incorporated into the RNA-induced silencing complex (RISC). Within the RISC, the miRNA serves as a guide to direct Argonaute-2 (Ago2) to target messenger RNAs (mRNA). The miRNA works by forming Watson-Crick base pairs with complementary sequences of the mRNA target. This mainly occurs within the $3^{\prime}$ untranslated region (UTR), but binding to the $5^{\prime}$ UTR or coding sequence is also possible. Reduction in protein levels are predominantly due to the miRNA promoting degradation of the mRNA target, with the reminder accounted for by translation inhibition (Bartel 2009, Krol, et al. 2010).
\end{abstract}

\section{Multi-targeting effects of miRNAs on pathways}

Although there are only $\sim 1500$ miRNAs in the human genome these are predicted to regulate a third or more of all protein-coding genes. This is because a single miRNA is typically able to target 200 mRNAs. RNA sequencing, ribosomal profiling (sequencing mRNAs during translation by ribosomes) and quantitative proteomics have provided critical insight into the impact of miRNA on protein levels in cells; overexpression or silencing of a miRNA produces changes to hundreds of proteins (Guo, et al. 2010, Selbach, et al. 2008).

Correspondence: David C. Henshall, Ph.D. Department of Physiology and Medical Physics Royal College of Surgeons in Ireland 123 St Stephen's Green, Dublin 2, IRELAND Ph: +35314028629 Fx: +3531402 2447 dhenshall@ rcsi.ie.

Disclosure The author has no conflicts of interest to declare. The author confirms that he has read the Journal's position on issues involved in ethical publication and affirm that this report is consistent with those guidelines. 


\section{miRNA inhibitors}

One promising approach to manipulating miRNAs is via chemically-modified antisense oligonucleotides. Termed "antagomirs", these bind the miRNA resulting in its depletion from the cell. Locked nucleic acid (LNA) modification of these oligonucleotides confers long-lasting stability in tissues and biofluids. As a result, LNA-antagomirs can produce miRNA silencing lasting many weeks and are associated with upregulation of large sets ( 200) of target mRNAs (Elmen, et al. 2008). A further development has been the design of so-called "tiny LNAs". These are 8-mer LNA oligonucleotides that target shared seed regions of miRNA families, thus producing more extensive silencing of miRNA functions (Obad, et al. 2011). A miRNA-based therapeutic, miravirsen - an LNA-based antagomir that targets miR-122 for the treatment of Hepatitis C - recently successfully completed Phase 2 clinical trials, suggesting manipulation of miRNA can be safe and tolerated in patients. MiRNAs may have unprecedented potential as future treatments of human diseases.

\section{Status epilepticus produces select changes to miRNA levels in the brain}

In 2010, the first research showing miRNAs were altered by status epilepticus appeared. Along with subsequent profiling studies, we now have a detailed understanding of the bidirectional spatio-temporal miRNA changes that accompany status epilepticus. Several display consistent change, including miR-34a, miR-132, miR-134 and miR-146a. Some anticorrelated expression between miRNA and mRNA profiles has been noted, consistent with the current model of how miRNAs mainly act to down-regulate their targets. The development of antibodies suitable for Ago2 immunoprecipitation enabled RISC-loaded miRNAs to be identified after status epilepticus, including miR-132 and miR-134 (JimenezMateos, et al. 2011; 2012). Nevertheless, we currently have a poor understanding of which proteins are actually being controlled by miRNAs after status epilepticus. Future efforts must also focus on identifying the mRNAs within the RISC.

\section{Effects of antagomirs on status epilepticus, seizure-induced neuronal death and epileptogenesis}

A highly attractive quality of miRNA-based drugs is the long-lasting effects achieved; hippocampal miRNAs can be inhibited for more than one month after a single intracerebral antagomir injection (Jimenez-Mateos, et al. 2012). To date, antagomirs have been used to target four miRNAs in vivo, including miR-34a, miR-132 and miR-134. Of these, inhibition of miR-34a and miR-132 was reported to reduce seizure-induced neuronal death but no effects were found on seizure severity or duration during status epilepticus (JimenezMateos, et al. 2011; Hu, et al. 2012). Silencing miR-134 produced the most remarkable effects. This miRNA is known to target proteins involved in the control of dendritic morphology, and thus potentially of direct relevance to excitatory neurotransmission. We showed that pre-treatment $(24 \mathrm{~h})$ with a single intracerebroventricular microinjection of LNA-antagomirs targeting miR-134 reduced kainate-induced status epilepticus by 50-70\% (Jimenez-Mateos, et al. 2012). Damage to the hippocampus was also strongly reduced in the mice pre-treated with the antagomirs. Of potential importance as a future anti-epileptogenic strategy, injection of antagomirs after status epilepticus reduced the later occurrence of spontaneous seizures by $90 \%$ or more (Figure 1). Silencing miR-134 also reduced progressive damage to the hippocampus. 


\section{Acknowledgments}

The author would like to thank Eva M. Jimenez-Mateos for assistance with the preparation of the manuscript. The author also acknowledges funding support from Science Foundation Ireland grants 08/IN.1/B1875 and 11/TIDA/ B1988, and National Institutes of Health grant R56 073714.

\section{References}

Bartel DP. MicroRNAs: target recognition and regulatory functions. Cell. 2009; 136:215-233. [PubMed: 19167326]

Elmen J, Lindow M, Silahtaroglu A, Bak M, Christensen M, Lind-Thomsen A, Hedtjarn M, Hansen JB, Hansen HF, Straarup EM, McCullagh K, Kearney P, Kauppinen S. Antagonism of microRNA-122 in mice by systemically administered LNA-antimiR leads to up-regulation of a large set of predicted target mRNAs in the liver. Nucleic Acids Res. 2008; 36:1153-1162. [PubMed: 18158304]

Guo H, Ingolia NT, Weissman JS, Bartel DP. Mammalian microRNAs predominantly act to decrease target mRNA levels. Nature. 2010; 466:835-840. [PubMed: 20703300]

Hu K, Xie YY, Zhang C, Ouyang DS, Long HY, Sun DN, Long LL, Feng L, Li Y, Xiao B. MicroRNA expression profile of the hippocampus in a rat model of temporal lobe epilepsy and miR-34atargeted neuroprotection against hippocampal neurone cell apoptosis post-status epilepticus. BMC Neurosci. 2012; 13:115. [PubMed: 22998082]

Jimenez-Mateos EM, Bray I, Sanz-Rodriguez A, Engel T, McKiernan R, Mouri G, Tanaka K, Sano T, Saugstad JA, Simon RP, Stallings RL, Henshall DC. MicroRNA expression profile after status epilepticus and hippocampal neuroprotection by targeting miR-132. Am J Pathol. 2011; 179:25192532. [PubMed: 21945804]

Jimenez-Mateos EM, Engel T, Merino-Serrais P, McKiernan RC, Tanaka K, Mouri G, Sano T, O'Tuathaigh C, Waddington JL, Prenter S, Delanty N, Farrell MA, O'Brien DF, Conroy RM, Stallings RL, Defelipe J, Henshall DC. Silencing microRNA-134 produces neuroprotective and prolonged seizure-suppressive effects. Nat Med. 2012; 18:1087-1094. [PubMed: 22683779]

Krol J, Loedige I, Filipowicz W. The widespread regulation of microRNA biogenesis, function and decay. Nat Rev Genet. 2010; 11:597-610. [PubMed: 20661255]

Obad S, dos Santos CO, Petri A, Heidenblad M, Broom O, Ruse C, Fu C, Lindow M, Stenvang J, Straarup EM, Hansen HF, Koch T, Pappin D, Hannon GJ, Kauppinen S. Silencing of microRNA families by seed-targeting tiny LNAs. Nat Genet. 2011; 43:371-378. [PubMed: 21423181]

Selbach M, Schwanhausser B, Thierfelder N, Fang Z, Khanin R, Rajewsky N. Widespread changes in protein synthesis induced by microRNAs. Nature. 2008; 455:58-63. [PubMed: 18668040] 


\section{Summary and future directions}

MiRNAs represent an entirely new target for the treatment and prevention of status epilepticus. Important next steps include optimizing doses, formulation and route of delivery. The overall safety of antagomirs and their effectiveness in other models must be established. Undoubtedly, other high-value miRNA targets for status epilepticus exist. Other tools for modulating miRNAs in vivo have also emerged including microRNA "sponges" which may provide even greater capacity to control miRNAs and influence status epilepticus and its harmful effects on the brain. 

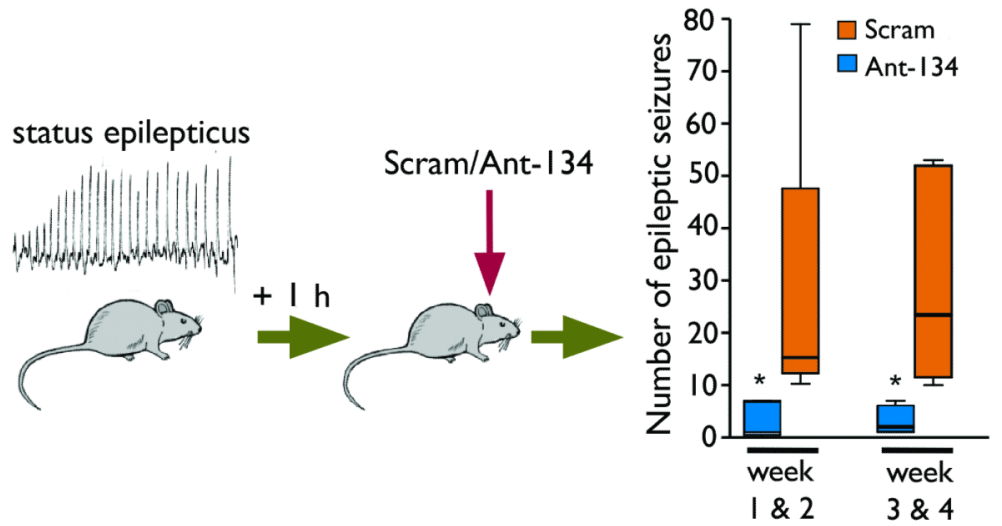

Figure 1.

Post-treatment with antagomirs reduces later spontaneous seizures. Mice were injected with antagomirs targeting miR-134 (Ant-134) or a non-targeting scrambled control (Scram) $1 \mathrm{~h}$ after triggering status epilepticus and then epilepsy monitoring commenced. Graph shows box-and-whisker plots of data on telemetry (weeks 1-2) and video-monitored (weeks 3-4) spontaneous seizures in mice $\left(n=5-6\right.$ per group; $\left.{ }^{*} p<0.05\right)$. Data are adapted from JimenezMateos et al. (2012) 